\title{
Colinesterasas en sangre total medidas con técnica semicuantitativa y en eritrocitos o plasma medidas con técnicas cuantitativas: relaciones
}

\author{
Jaime Carmona-Fonseca \\ Grupo Malaria, Facultad de Medicina, Universidad de Antioquia, Medellín, Colombia
}

Introducción. Se necesita un modelo que permita convertir valores de colinesterasa obtenidos con la técnica Lovibond ${ }^{\circledR}$ (semicuantitativa para sangre total) en cifras equivalentes en las técnicas Michel, $\mathrm{EQM}^{\circledR}$ y Monotest ${ }^{\circledR}$ (cuantitativas eritrocitarias y plasmáticas).

Objetivo. Examinar la relación entre Lovibond ${ }^{\circledR}$ (técnicas tintométrica de Edson y de Limperos y Ranta) y las técnicas cuantitativas.

Materiales y métodos. Diseño descriptivo, transversal, prospectivo, con dos muestras representativas de poblaciones laborales de 18 a 59 años, sin exposición a plaguicidas inhibidores de colinesterasa, vinculadas al Seguro Social en el valle de Aburrá y en el cercano oriente antioqueño.

Resultados. Se evaluaron 827 trabajadores. Según la técnica Lovibond ${ }^{\circledR}$ se clasificaron en cuatro categorías: 821 con valores de $75 \%$ o más de actividad colinesterásica (categorías $75 \%, 87,5 \%$ y $100 \%$ ) y otros seis con actividad inferior a $75 \%$. Con cada técnica cuantitativa los valores promedios de colinesterasa eritrocitaria y plasmática correspondientes a los cuatro valores de Lovibond ${ }^{\circledR}$ fueron estadísticamente diferentes entre sí. Los promedios de cada técnica cuantitativa fueron mayores a medida que creció el valor de la técnica semicuantitativa. Con Lovibond ${ }^{\circledR}$ se clasificó muy mal $(61 \%-73 \%)$ la baja actividad enzimática eritrocitaria, pero la clasificación de la baja actividad plasmática fue absolutamente errónea (94\%-96\%).

Conclusión. Es posible estimar en forma adecuada los valores de colinesterasa eritrocitaria o plasmática que corresponden a las técnicas de Michel y $\mathrm{EQM}^{\circledR}$ a partir de datos de la técnica Lovibond ${ }^{\circledR}$ y viceversa, pero sólo cuando la actividad colinesterásica es normal, pues Lovibond ${ }^{\circledR}$ tiene una capacidad muy deficiente para identificar como "bajos" los valores que realmente lo son según las pruebas cuantitativas.

Palabras clave: inhibidores de la colinesterasa, acetilcolinesterasa, butirilcolinesterasa, plaguicidas, compuestos organofosforados, vigilancia epidemiológica, Colombia

Cholinesterases in total blood measured with a semiquantitative technique, and plasma or erythrocyte cholinesterases measured with quantitative techniques

Introduction. An equivalence model which allows comparison of blood cholinesterase values, measured by Lovibond ${ }^{\circledR}$ (semiquantitative technique), and Michel, EQM ${ }^{\circledR}$, Monotest ${ }^{\circledR}$ (erythrocyte and plasma cholinesterases) values measured by quantitative techniques is required.

Objective. The performance of Lovibond ${ }^{\circledR}$ (Edson tintometric and Limperos \& Ranta techniques) were compared with quantitative techniques.

Materials and methods. The experimental design was descriptive, cross-sectional, and prospective. From a working population (18-59 years) in Valle de Aburrá and Near East of Antioquia. 827 representative samples were chosen for their lack of exposure to cholinesteraseinhibiting plaguicides and affiliated to the Social Security System.

Results. (1) 827 workers were classified by Lovibond ${ }^{\circledR}$ in four categories: 821 values with $75 \%$ of cholinesterase activity or greater (categories 75, 87.5 and 100\%) and 6 with cholinesterase activity smaller than $75 \%$. (2) With each quantitative method, the mean values of erythrocyte and plasmatic cholinesterase corresponding to the four values obtained with Lovibond® were statistically different to each other. (3) The mean values of each quantitative technique increased 
when increased the tintometric method value. (4) Lovibond® classified the low enzymatic erythrocyte activity very poorly $(61-73 \%)$, but the classification of the low enzymatic plasma activity was almost completely in error (94-96\%).

Conclusion. The values of erythrocyte or plasma cholinesterase were adequately estimated by both the quantitative techniques of Michel and EQM ${ }^{\circledR}$ and by Lovibond, but only when the enzymatic activity is normal. Lovibond ${ }^{\circledR}$, however, had a poor capacity to designate as "low" the values that were low according to the quantitative tests.

Key words: Cholinesterase inhibitors, acetylcholinesterase, butyrylcholinesterase, pesticides, organophosphorus compounds, epidemiological surveillance, Colombia

En Colombia los valores de referencia de la colinesterasa han sido tomados de estudios en países con características de población muy diferentes. Tales estándares han surgido de grupos muy pequeños no representativos de la población. En el decenio 1990 a 1999, el Instituto de Seguros Sociales (ISS) adelantó varios trabajos de investigación tendientes a definir los valores autóctonos de colinesterasa en la población laboral colombiana. En primer lugar, se estandarizaron varios métodos y se midió la actividad de colinesterasa en niños trabajadores (14-17 años) (1). Después, a partir de muestras representativas de la población laboral adulta no expuesta a plaguicidas inhibidores de colinesterasa y afiliada al ISS en empresas del valle de Aburrá y del cercano oriente antioqueño, se midió la actividad colinesterásica en sangre total, en eritrocitos y en plasma mediante seis técnicas diferentes:

a) Sangre total: técnica de Limperos y Ranta, luego modificada por Edson, conocida también como técnica tintométrica o colorimétrica de Edson; se usó el estuche producido por Lovibond $^{\circledR}$ (2-4), un procedimiento semicuantitativo que expresa valores de actividad enzimática en nueve intervalos de $12,5 \%$ cada uno, correspondientes a una actividad de $100 \%$, $87,5 \%, 75 \%$ y así sucesivamente, con respecto a un control no expuesto a plaguicidas inhibidores de colinesterasa (5).

b) Eritrocitos: técnicas cuantitativas de Michel (técnica electrométrica, que es el patrón o

\footnotetext{
Correspondencia:

Jaime Carmona-Fonseca, Grupo Malaria, Facultad de Medicina, Universidad de Antioquia, calle 62 No. 52-59, laboratorio 610, Medellín, Colombia

jaimecarmonaf@hotmail.com
}

Recibido: 06/12/06; aceptado: 27/03/07 referencia) $(6,7)$ y EQM ${ }^{\circledR}(8-10)$, basada en el método espectrofotométrico-colorimétrico de Ellman, modificado (11).

c) Plasma: técnicas cuantitativas de Michel, $\mathrm{EQM}^{\circledR}$ y Monotest ${ }^{\circledR}$ (12), esta última basada en un método cinético y producida por Boehringer Mannheim (13) (cuadro 1).

d) Se identificaron también valores de colinesterasa en varios grupos de mujeres trabajadoras según su estado hormonal sexual (14).

e) Se evaluó el comportamiento de la enzima en enfermos crónicos controlados, teniendo en cuenta el sexo, la zona de residencia, el uso de medicamentos y otras variables (15).

f) De igual manera, se conocieron los valores de cianometahemoglobina y hematocrito de esas poblaciones (16).

g) Se identificó la frecuencia de grupos sanguíneos $A B O$ y $R h$ en la población laboral (17) y la relación entre estos grupos y la actividad de la colinesterasa (18).

h) Se establecieron las correlaciones matemáticas entre los valores de colinesterasa en eritrocitos medidos por las dos técnicas cuantitativas (Michel y $\mathrm{EQM}^{\circledR}$ ) (19) y, en plasma, medidos por las tres técnicas cuantitativas (CarmonaFonseca, en proceso de publicación).

El programa colombiano de vigilancia epidemiológica de plaguicidas organofosforados y carbamatos (Programa VEO) opera desde 1982 y lo hace por un convenio entre el Laboratorio de Salud Ambiental del Instituto Nacional de Salud y las direcciones seccionales de salud del país. De 1993 a 2001 , entre $6,1 \%$ y $7,6 \%$ de las muestras examinadas a los trabajadores colombianos 
Cuadro 1. Valores de referencia para cada técnica de medición obtenidos en el estudio de colinesterasas de población laboral; valle de Aburrá y cercano oriente de Antioquia $(5,11,13)$

\begin{tabular}{|c|c|c|c|c|c|c|}
\hline Técnica (1) & Sexo & Promedio & DE & EE & LI-IC95\%X & LS-IC95\%X \\
\hline \multicolumn{7}{|l|}{ Eritrocitos } \\
\hline \multirow[t]{3}{*}{ Michel } & general & 0,857 & 0,127 & 0,00442 & 0,849 & 0,866 \\
\hline & hombres & 0,868 & 0,185 & 0,00643 & 0,855 & 0,881 \\
\hline & mujeres & 0,847 & 0,175 & 0,00607 & 0,836 & 0,869 \\
\hline \multirow{3}{*}{ EQM } & general & 35,21 & 5,74 & 0,19946 & 34,82 & 35,6 \\
\hline & hombres & 35,1 & 8,50 & 0,29565 & 34,52 & 36,68 \\
\hline & mujeres & 35,33 & 7,70 & 0,26781 & 34,8 & 35,85 \\
\hline \multicolumn{7}{|l|}{ Plasma } \\
\hline \multirow[t]{3}{*}{ Michel } & general & 1,118 & 0,261 & 0,00908 & 1,1 & 1,136 \\
\hline & hombres & 1,169 & 0,387 & 0,01346 & 1,142 & 1,195 \\
\hline & mujeres & 1,067 & 0,351 & 0,01219 & 1,043 & 1,091 \\
\hline \multirow[t]{3}{*}{ EQM } & general & 2,512 & 0,62 & 0,02154 & 2,469 & 2,554 \\
\hline & hombres & 2,572 & 0,92 & 0,03192 & 2,509 & 2,635 \\
\hline & mujeres & 2,451 & 0,83 & 0,02892 & 2,394 & 2,508 \\
\hline \multirow[t]{3}{*}{ Monotest } & general & 5.601 & 1.611 & 56 & 5.491 & 5711 \\
\hline & hombres & 5.822 & 2.387 & 83 & 5.659 & 5985 \\
\hline & mujeres & 5.380 & 2.157 & 75 & 5.232 & 5228 \\
\hline \multicolumn{7}{|l|}{ Sangre total } \\
\hline \multirow[t]{3}{*}{ Lovibond } & general & 92 & 12 & 0,4 & 91 & 93 \\
\hline & hombres & 94 & 17 & 0,6 & 93 & 95 \\
\hline & mujeres & 90 & 17 & 0,6 & 89 & 91 \\
\hline
\end{tabular}

(1) Las unidades de medida de las técnicas son: Michel deltas pH/hora, EQM ${ }^{\circledR} \mathrm{U} / \mathrm{g}$ oxihemoglobina, Monotest ${ }^{\circledR} \mathrm{U} / \mathrm{L}$ y Lovibond ${ }^{\circledR}$ porcentaje.

expuestos a plaguicidas inhibidores de colinesterasa y cubiertos por el programa de vigilancia epidemiológica, tenían valores bajos de colinesterasa en sangre total (20-22); en los departamentos de Córdoba y Bolívar el porcentaje fue muy alto: $17,7 \%$ y $20,3 \%$, en su orden, lo que implicaría que uno de cada cinco a seis expuestos estaba intoxicado, situación más grave si se conoce que apenas $36 \%$ de los estudiados tenían afiliación a la seguridad social (21).

Los autores del informe de 2005 del Programa VEO afirmaron que la diversidad de pesticidas usados plantea la necesidad de aumentar la variedad de biomarcadores analizados para vigilar la exposición (22), lo cual ya había sido señalado antes, cuando se dijo que en Colombia "la exposición ha cambiado en los últimos 50 años; se pasó del uso de organoclorados y compuestos arsenicales a una amplísima variedad de plaguicidas entre los que sobresalen, últimamente, los ditiocarbamatos" (23); también se afirmó que "debe revisarse la forma en la cual se está manejando el Programa VEO y fortalecerlo en aquellas regiones donde existen poblaciones con clara exposición a plaguicidas inhibidores de la colinesterasa" (23).

El Programa VEO usa el estuche Lovibond ${ }^{\circledR}$ que mide la actividad enzimática en sangre total (eritrocitos y plasma) (24). En 1994, McConnell y Magnotti reevaluaron esta técnica (25) y señalaron que la prueba tintométrica de campo que se emplea en "los países subdesarrollados es económica" y que cuando Miller y Shah, en 1982, la calificaron como adecuada, hubo problemas, por lo que debía ser revalorada; Miller y Shah habían concluido que las diferencias con respecto a una prueba estándar "no fueron más allá de aquellas que pueden esperarse como inherentes al método" (26), pero McConnell y Magnotti anotaron que "sin embargo, la sensibilidad y la especificidad del estuche y su precisión para identificar trabajadores con colinesterasa eritrocitaria levemente reducida no fueron presentadas" en la evaluación de 1982 (25). 
Esos autores reevaluaron, entonces, el estuche tintométrico y para ello usaron como prueba de referencia una que era modificación del método estándar de Ellman y colaboradores (27), prueba espectrofotométrica-colorimétrica que luego fue patentada como $\mathrm{EQM}^{\circledR}$ (EQM Research Inc., Cincinnati, Ohio, EUA); según dijeron McConnell y Magnotti, el estándar que usaron estaba adaptado como prueba de campo para medir colinesterasa eritrocitaria y su validez había sido establecida en población normal y expuesta a plaguicidas inhibidores de colinesterasa, tanto con prueba en laboratorio como de campo $(9,10,28)$. Concluyeron que "aunque la correlación global entre la prueba de referencia (espectrofotométrica) y la prueba tintométrica fue buena, tanto la sensibilidad como la especificidad fueron menores de $75 \%$ para cada uno de los tres valores críticos de la prueba tintométrica comúnmente usados para definir anormalidad $(75 \%, 62,5 \%$ y $50 \%$ del control). Además, aunque hubo buena concordancia entre los dos métodos en los valores menores de $50 \%$ de actividad del control, en las categorías de $87,5 \%$ y $100 \%$ hubo 3 de 29 muestras con baja actividad según la prueba de referencia. Según el fabricante, esas muestras deberían ser normales. Esos resultados concuerdan con nuestra previa observación de que el estuche tintométrico sobreestima sustancialmente la actividad colinesterásica durante la fase de recuperación del envenenamiento" (25), según lo habían informado (10).

Se ha dicho también que Lovibond ${ }^{\circledR}$ "es una técnica simple, barata y de fácil uso en terreno. Sin embargo, esta técnica es poco exacta y su calidad depende de dos factores de difícil control como son la temperatura del ensayo y la habilidad del operador. Por esto, su uso en el marco de esta evaluación resulta limitado, aunque sigue siendo válida para confirmar intoxicación clínica por órganofosforados y/o carbamatos" (29); otros han tenido menos objeciones y afirmaron que "es un método de laboratorio muy sencillo, que permite detectar en terreno los niveles de colinesterasa en trabajadores expuestos a plaguicidas organofosforados, además de demostrar rendimiento por costo-beneficio, entrega de resultados casi de forma inmediata (una hora como promedio), lo cual depende de variables ambientales como la temperatura y de no requerir de personal especializado" (Sancliment-Montaño R, Olivo-Garza J, Moreno-Álvarez M. Reunión Nacional de Investigación de Seguridad e Higiene. Monitoreo biológico de trabajadores expuestos a plaguicidas organofosforados).

En 1994 se propuso cambiar el disco comparador del equipo Lovibond ${ }^{\circledR}$ por una solución estándar que tenga colores similares, porque el uso de soluciones estándares coloreadas parece más apropiado para las condiciones de campo (30); los autores usaron diluciones de azul de bromotimol, sangre total y ácido acético en diferentes concentraciones para construir un conjunto de soluciones coloreadas a las cuales correspondieron diferentes grados de inhibición de la colinesterasa eritrocitaria; midieron la actividad enzimática con los dos procedimientos (Lovibond ${ }^{\circledR}$ con disco y soluciones) y hallaron buena concordancia y reproducibilidad satisfactoria de los resultados. También se ha comparado la técnica Lovibond ${ }^{\circledR}$, que usa muestras frescas, con otra que emplea sangre congelada (técnica de Oliveira-Silva) (31).

Nosotros hallamos una correlación significativa entre las dos técnicas cuantitativas eritrocitarias (Michel y $\mathrm{EQM}^{\circledR}$ ): de 827 trabajadores, el coeficiente " $r$ " fue 0,666 y el coeficiente $R^{2}$ fue $44 \%$; el análisis de varianza demostró que los coeficientes "a" (intersección) y "b" (pendiente) eran muy significativos ( $p=0,00000)$; el coeficiente " $r$ " se elevó a 0,718 con el hecho de eliminar doce de los valores extremos y $\mathrm{R}^{2}$ pasó a $51 \%$ (19). Entre las tres pruebas cuantitativas para colinesterasa plasmática (Michel $\mathrm{EQM}^{\circledR}$, Monotest $\left.{ }^{\circledR}\right)$ también se encontró correlación significativa (Carmona-Fonseca, en proceso de publicar).

El presente artículo da cuenta de un análisis cuyo objetivo fue explorar la relación entre la colinesterasa en sangre total medida con la prueba tintométrica semicuantitativa de Lovibond ${ }^{\circledR}$ y la colinesterasa eritrocitaria o plasmática medida con las técnicas cuantitativas (Michel, EQM ${ }^{\circledR}$, Monotest ${ }^{\circledR}$ ) en parte de la población laboral colombiana, con el fin de facilitar la expresión de 
las mediciones por un método en los valores correspondientes a los otros, porque en el país y en otros lugares se usan procedimientos diferentes con fines epidemiológicos y clínicos. Los resultados básicos con cada una de las técnicas se han informado antes y con detalle $(5,11,13)$; ahora el trabajo se limita al análisis de las relaciones entre la técnica semicuantitativa y las técnicas cuantitativas.

\section{Materiales y métodos}

Diseño de la muestra de población, recolección de información, criterios de inclusión en el estudio

Los detalles metodológicos del estudio se publicaron antes $(5,11,13)$. Se empleó un diseño descriptivo, transversal y prospectivo. Se tomaron sendas muestras representativas de la población laboral adulta, de 18 a 59 años, sin exposición a plaguicidas inhibidores de colinesterasa, vinculada a empresas afiliadas al Seguro Social y situadas en el valle de Aburrá y en el cercano oriente antioqueño. Además, y sólo para fines complementarios, se incluyeron personas de 60 a 75 años, que eran trabajadores activos.

Se investigó la presencia de enfermedades que alteran los niveles de colinesterasas y quien tenía alguna de ellas se excluyó del estudio. Los estados fisiológicos como embarazo y menstruación, que modifican los valores de actividad de colinesterasa, no fueron causa de exclusión. Tampoco lo fue la ingestión de medicamentos si el trabajador decía sentirse bien, pero quienes dijeron tomarlos y afirmaron no estar con buena salud sí fueron dejados por fuera del estudio.

\section{Técnicas de colinesterasa}

A cada trabajador se le realizaron mediciones de la actividad de colinesterasa en sangre total $\left(\right.$ Lovibond $^{\circledR}$ ), en eritrocitos (Michel, EQM ${ }^{\circledR}$ ) y en plasma (Michel, EQM ${ }^{\circledR}$, Monotest ${ }^{\circledR}$ ). Cada muestra fue analizada una vez. Las técnicas empleadas están basadas en los principios de a) la medición del ácido producido de la acetilcolina, acetilbeta-metilcolina o de la butirilcolina; b) la medición de la hidrólisis de la tiocolina $(1,5,11,13)$. Las pruebas fueron ejecutadas en laboratorios que ya las tenían estandarizadas y que aplican programas de control de calidad.

\section{Análisis estadístico}

El análisis se hizo con los programas Epilnfo 6.00, SGPlus 7.1 y SPSS 10.0. Se realizó un análisis de varianza no paramétrico de una vía (prueba de Kruskal y Wallis) para evaluar la variación de cada una de las variables cuantitativas (Michel, EQM ${ }^{\circledR}$, Monotest ${ }^{\circledR}$ ) en función de los valores de la variable ordinal Lovibond ${ }^{\circledR}$, que expresa los niveles de colinesterasa sanguínea en intervalos de $12,5 \%$ entre $0 \%$ y $100 \%$. Se usó el procedimiento de análisis de rango múltiple de Newman y Keuls con un nivel de confianza de $95 \%$ para comparar pares de medias.

Se usan estas expresiones: DE: desviación estándar; EE: error estándar; n: número de personas (tamaño del grupo); IC95\%: intervalo de confianza de 95\%; anova: análisis de varianza, KW estadístico de Kruskal y Wallis; $\chi^{2}$ : prueba de ji al cuadrado; Fisher: prueba de ji al cuadrado exacta de Fisher; Bartlett: estadístico de Bartlett; ARM Newman-Keuls: análisis de rangos múltiples de Newman y Keuls.

\section{Resultados}

La distribución de cada conjunto de datos de las pruebas de colinesterasa cuantitativas fue normal (gaussiana), según la estadística descriptiva, el histograma de frecuencias y la prueba para normalidad de Kolmogorov-Smirnov.

Se hizo un análisis que compara las colinesterasas eritrocitaria y sanguínea y, por otra parte, la plasmática con la sanguínea. Este análisis no puede hacerse mediante correlación-regresión porque Lovibond ${ }^{\circledR}$ usa una escala discreta (no es continua). El estudio se hizo por medio de la prueba de Kruskal y Wallis (KW); se compararon los cuatro valores de actividad enzimática de la variable cuantitativa correspondientes a los cuatro valores (categorías) de la prueba tintométrica $(<75 \%, 75 \%, 82,5 \%$ y $100 \%)$, para responder la pregunta ¿son iguales los tres promedios? Luego, con un análisis de rango múltiple por el método de Newman y Keuls, se identificaron los pares de promedios que difieren estadísticamente entre sí. 
Si esas comparaciones por KW y el ARM muestran que entre los cuatro valores comparados hay diferencia significativa y si, además, los promedios de una técnica cuantitativa en función de Lovibond ${ }^{\circledR}$ presentan alguna tendencia positiva (cuando la cuantitativa aumenta también lo hace la discreta), puede pensarse que si la medición con la técnica tintométrica fuese una variable continua se podría estar frente a una situación de correlación-regresión entre la técnica cuantitativa y Lovibond ${ }^{\circledR}$. El análisis de varianza de KW mostró que (cuadro 2, figura 1):

a) con cada técnica cuantitativa, hubo diferencia estadísticamente significativa entre los valores promedios de colinesterasa eritrocitaria y plasmática correspondientes a los cuatro valores de Lovibond ${ }^{\circledR}$;

b) con cada técnica cuantitativa, los promedios fueron mayores a medida que creció el valor de la prueba tintométrica, y

c) en cada técnica cuantitativa, los valores promedios difirieron entre sí, según el ARM de Newman-Keuls, es decir, todos difieren entre sí.
Según esos resultados, para un valor de 100\% de actividad enzimática según la técnica tintométrica corresponden, en eritrocitos, 0,883 deltas $\mathrm{pH} /$ hora con la técnica de Michel y 35,98 $\mathrm{U} / \mathrm{g}$ oxihemoglobina con $\mathrm{EQM}^{\circledR}$. Ambos promedios están por fuera del IC95\% para el promedio calculado directamente con los 827 datos, intervalos que fueron 0,849 a 0,866 con Michel (promedio $=0,857$ ) y 34,82 a $35,60 \mathrm{U} / \mathrm{g}$ oxihemoglobina con EQM ${ }^{\circledR}$ (promedio=35,21). De igual forma, un valor de $100 \%$ de actividad enzimática según la técnica tintométrica corresponde, en plasma, a 1,172 deltas $\mathrm{pH} /$ hora con la técnica de Michel, a 2,642 U/g oxihemoglobina con EQM ${ }^{\circledR}$ y a $5943 \mathrm{U} / \mathrm{L}$ con Monotest ${ }^{\circledR}$. Aquí también los tres promedios están por fuera del IC95\% para el promedio calculado directamente con los 827 datos, intervalos que fueron 1,100 a 1,136 con Michel (promedio: 1,118); 2,469 a 2,554 U/g oxihemoglobina con $\mathrm{EQM}^{\circledR}$ (promedio $=2,512$ ) y 5.491 a 5.711 con Monotest $^{\oplus}$ (promedio=5.601).

¿Cómo fue el comportamiento entre las técnicas al medir valores bajos de actividad enzimática? En otras palabras, ¿cómo fue la concordancia entre

Cuadro 2. Valores de colinesterasa eritrocitaria y plasmática medida con una técnica cuantitativa en función de los valores de la técnica semicuantitativa Lovibond ${ }^{\circledR}$

\begin{tabular}{|c|c|c|c|c|c|c|c|c|c|c|c|c|c|}
\hline $\begin{array}{l}\text { Eritrocitos } \\
\text { Lovibond }\end{array}$ & $\begin{array}{c}s(1) \\
n\end{array}$ & $\begin{array}{l}\text { Media } \\
\text { Michel }\end{array}$ & DE & IC95\% & Media & $\begin{array}{c}\text { Media } \\
\text { EQM }^{\circledR}\end{array}$ & DE & IC95\% & Media & & & & \\
\hline$<75,0$ & 6 & 0,750 & 0,055 & 0,6916 & 0,7984 & 32,17 & 3,09 & 28,92 & 35,41 & & & & \\
\hline 75,0 & 85 & 0,795 & 0,079 & 0,7841 & 0,8182 & 33,17 & 3,77 & 32,36 & 33,99 & & & & \\
\hline 87,5 & 421 & 0,836 & 0,079 & 0,8293 & 0,8444 & 34,48 & 3,83 & 34,12 & 34,85 & & & & \\
\hline 100,0 & 315 & 0,883 & 0,091 & 0,8738 & 0,8929 & 35,98 & 3,65 & 35,58 & 36,39 & & & & \\
\hline total & 827 & 0,850 & 0,086 & 0,8443 & 0,8561 & 34,90 & 3,86 & 34,64 & 35,17 & & & & \\
\hline $\begin{array}{l}\text { Plasma (1) } \\
\text { Lovibond }\end{array}$ & 1) $n$ & $\begin{array}{l}\text { Media } \\
\text { Michel }\end{array}$ & DE & IC95\% & Media & $\begin{array}{c}\text { Media } \\
\text { EQM }^{\circledR}\end{array}$ & DE & IC95\% & $\underset{\mathbf{N}}{\text { Media }}$ & $\begin{array}{c}\text { Media } \\
\text { Monotest }\end{array}$ & DE & IC95\% & Media \\
\hline$<75,0$ & 6 & 0,733 & 0,121 & 0,6430 & 0,8433 & 1,600 & 0,190 & 1,4244 & 1,7789 & 3.146 & 726 & 2.384 & 3.908 \\
\hline 75,0 & 85 & 0,965 & 0,174 & 0,9275 & 0,9993 & 2,140 & 0,365 & 2,0551 & 2,2111 & 4.621 & 907 & 4.426 & 4.817 \\
\hline 87,5 & 421 & 1,056 & 0,176 & 1,0392 & 1,0723 & 2,362 & 0,371 & 2,3267 & 2,3973 & 5.253 & 1.017 & 5.156 & 5.351 \\
\hline 100,0 & 315 & 1,172 & 0,176 & 1,1557 & 1,1935 & 2,642 & 0,439 & 2,5921 & 2,6893 & 5.943 & 1.134 & 5.818 & 6.069 \\
\hline total & 827 & 1,089 & 0,187 & 1,0765 & 1,1020 & 2,439 & 0,434 & 2,4094 & 2,4688 & 5.436 & 1.159 & 5.357 & 5.514 \\
\hline
\end{tabular}

Conclusión: en cada técnica comparada con Lovibond: 1) los cuatro promedios difieren significativamente (siempre $\mathrm{p}=0,000000$ en la prueba de Kruskal-Wallis); 2) en cada par de comparaciones, los promedios tienen diferencia significativa (ARM Newman-Keuls); 3) siempre se observa una relación proporcional entre Lovibond y la técnica cuantitativa: si la primera aumenta, la cuantitativa también lo hace.

(1) Las unidades de medida de las técnicas son: Michel, deltas pH/hora; EQM ${ }^{\circledast}, \mathrm{U} / \mathrm{g}$ oxihemoglobina; Monotest ${ }^{\circledR}, \mathrm{U} / \mathrm{L}^{\mathrm{y}}$ Lovibond $^{\circledR}$, porcentaje. 


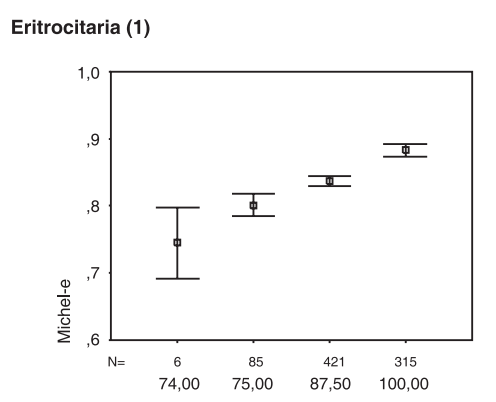

Lovibond

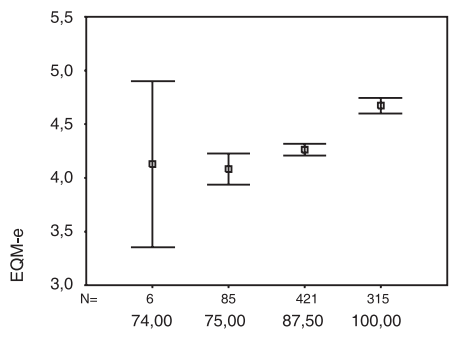

Lovibond

Plasmática (1)

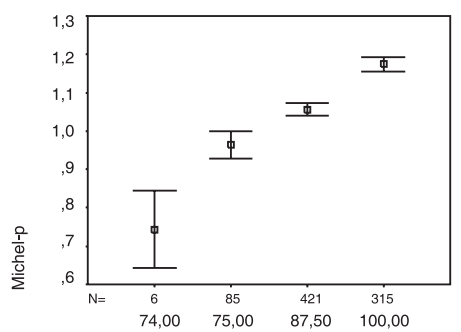

Lovibond

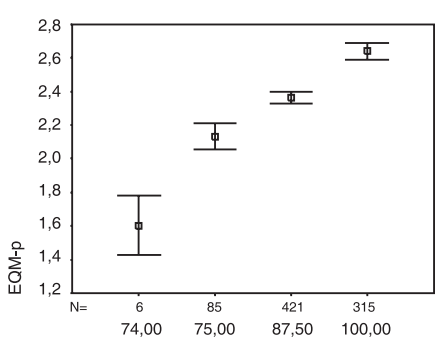

Lovibond

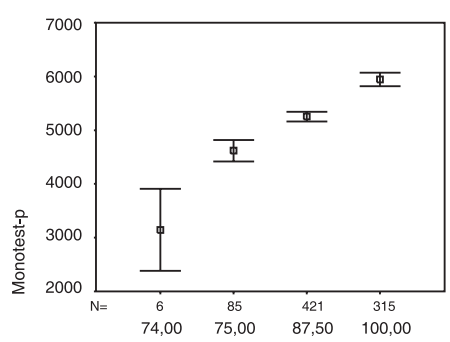

Lovibond

(1) El valor 74 de cada figura significa menor que 75 .

Figura 1. Variación de la colinesterasa medida cuantitativamente (Michel, EQM, Monotest) según cuatro valores fijos de la técnica semicuantitativa (Lovibond)

Unidades de medida: Michel, deltas pH/hora; $\mathrm{EQM}^{\circledast}, \mathrm{U} / \mathrm{g}$ oxihemoglobina; Monotest ${ }^{\circledR}, \mathrm{U} / \mathrm{L}^{\text {y }}$ Lovibond $^{\circledR}$, porcentaje

la clasificación de los resultados por una técnica cuantitativa y por el procedimiento semicuantitativo? Hubo seis personas con valores Lovibond ${ }^{\circledR}$ por debajo de $75 \%$ (uno con $50 \%$ y cinco con 62,7\%); se espera, entonces, que si la técnica tintométrica los clasifica como poseedores de baja actividad de colinesterasa, lo mismo haga la otra técnica y ello fue así para todos los seis sujetos en el caso de la enzima plasmática, pero no hubo concordancia en ninguna de las personas en el caso de la enzima eritrocitaria, porque las seis tenían valores superiores al que corresponde al $80 \%$ del límite inferior del IC95\% para el promedio.
La casa fabricante de Lovibond ${ }^{\circledR}$ recomienda una escala para interpretar los resultados del análisis, escala que dice que un resultado de $75 \%$ a $100 \%$ de la actividad del control normal no requiere aplicar ninguna acción a la persona; de $50 \%$ a $75 \%$ de actividad indica una probable sobreexposición, debe repetirse la prueba y, si se confirma el resultado, la persona (usualmente un trabajador) debe retirarse de la exposición por dos semanas; de $25 \%$ a $50 \%$ indica sobreexposición, debe repetirse la prueba y, si se confirma el resultado, la persona debe retirarse de la exposición y, si es indispensable, deben ordenarse exámenes; de $0 \%$ a $25 \%$ de lo normal indica muy 
seria y peligrosa sobreexposición, debe repetirse la prueba y, si se confirma el resultado, la persona debe retirarse de la exposición y someterse a examen médico. Esta escala tiene un error metodológico de fondo, consistente en que traslapa los intervalos, pues $75 \%, 50 \%$ y $25 \%$ hacen parte simultáneamente de dos intervalos. Para el análisis, se modificó tal clasificación y se usaron los intervalos 75\%-100\%, 50\%-62,5\% y menos de 50\%. El análisis llevó a concluir que la clasificación que hizo Lovibond ${ }^{\circledR}$ de las personas con baja actividad enzimática en eritrocitos fue muy deficiente (61\%-73\% mal clasificadas), pero la que hizo de la actividad plasmática fue absolutamente errónea (94\%-96\% mal clasificadas). Éste fue el resultado más importante del análisis: Lovibond ${ }^{\circledR}$ fue incapaz de clasificar como poseedoras de baja actividad a las personas cuya actividad enzimática eritrocitaria o plasmática estaba francamente reducida (porque estaban por debajo del valor que corresponde a $80 \%$ del límite inferior del intervalo normal, IC95\% para el promedio), de tal forma que, si se confiase en los resultados de Lovibond ${ }^{\oplus}$, el trabajador seguiría expuesto al plaguicida.

Por otra parte, en cuanto a la capacidad mostrada por Lovibond ${ }^{\circledR}$ para clasificar adecuadamente los valores de colinesterasa "altos", según las técnicas cuantitativas, encontramos lo siguiente, anotando que "alto" significó un valor mayor al límite superior del IC95\% para el promedio (cuadro 3):

a) En la enzima eritrocitaria, Lovibond ${ }^{\circledR}$ clasificó con $75 \%$ de la actividad a $3,7 \%$ de los valores de Michel y a 6,5\% de los correspondientes a $\mathrm{EQM}^{\circledR}$, donde se incluye un valor con $62,5 \%$ de actividad (que sería claramente bajo);

Cuadro 3. Aproximación a evaluar la capacidad diagnóstica de Lovibond ${ }^{\circledR}$ frente a la técnica patrón (Michel) y a otras dos técnicas cuantitativas $\left(\mathrm{EQM}^{\circledR}\right.$ y Monotest $\left.^{\circledR}\right)$.

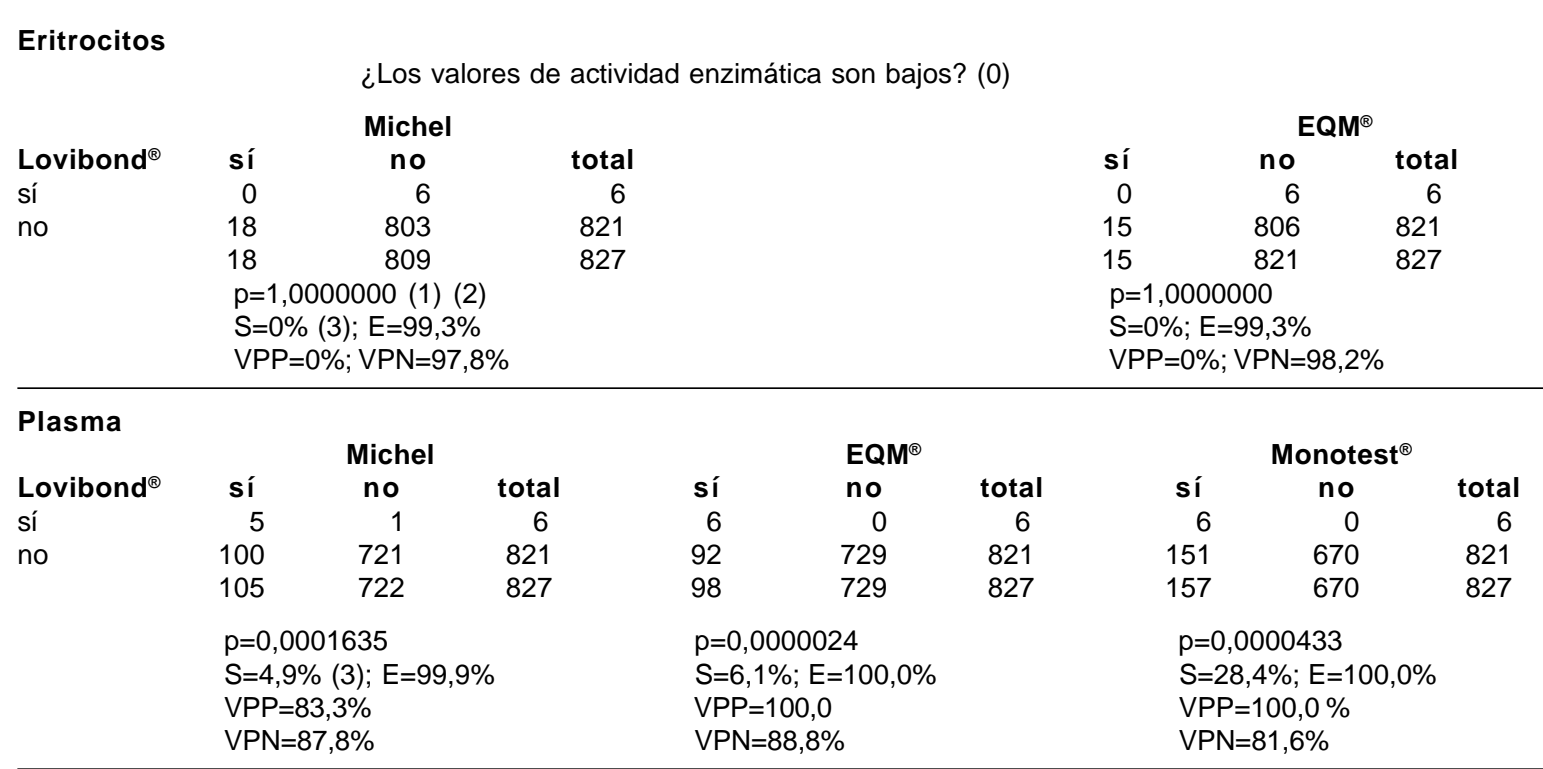

(0) En cada prueba "no" y "sí" se refieren a si los valores de actividad enzimática son bajos o no, habiendo definido "baja actividad" como menos del valor correspondiente a $80 \%$ del límite inferior del IC95\% para promedio en una técnica cuantitativa o $<75 \%$ en Lovibond ${ }^{\circledR}$.

(1) En todas las comparaciones, siempre hubo un valor esperado de $<5 \mathrm{y}$, por ello, debe usarse la prueba exacta de ji al cuadrado de Fisher. El valor $\mathrm{p}$ corresponde siempre al de 2 colas.

(2) Si el valor $p$ no es significativo, indica que las dos técnicas no difieren en la clasificación, es decir, que concuerdan. La concordancia ocurrió para Lovibond y cada ténica cuantitativa al medir la colinesterasa eritrocitaria, pero no hubo concordancia al medir la enzima plasmática.

(3) S: sensibilidad; E: especificidad; VPP: valor de predicción positivo; VPN: valor de predicción negativo 
b) En la enzima plasmática, Lovibond ${ }^{\circledR}$ clasificó con $75 \%$ de la actividad a $4,5 \%$ de los valores de Michel, a 3,1\% de los correspondientes a $E M^{\circledR}$ y a $2,2 \%$ de aquellos de Monotest ${ }^{\circledR}$.

Al clasificar los valores de cada técnica en "bajos" y "no bajos" y compararlas se tiene una aproximación a lo que sería una evaluación de la capacidad diagnóstica de Lovibond ${ }^{\circledR}$ frente a Michel y a las otras dos pruebas cuantitativas; el análisis permite apreciar que es nula la "sensibilidad" de la técnica tintométrica y muy alta su "especificidad" frente a Michel y EQM ${ }^{\otimes}$ para ambas colinesterasas (eritrocitaria y plasmática) y muy baja la "sensibilidad" (28\%) y muy alta la "especificidad" frente a Monotest ${ }^{\circledR}$, todo lo cual concuerda con los anteriores análisis. Esto indica que la técnica tintométrica no sería apropiada para tamizar.

\section{Discusión}

Hemos logrado obtener valores autóctonos para la población laboral adulta, no expuesta a plaguicidas inhibidores de colinesterasa, y vinculada al Seguro Social por medio de empresas de dos grandes áreas del departamento de Antioquia (cuadro 1). Para la técnica de Michel, esos valores nuestros difieren notoriamente de otros presentados por varios autores (cuadro 4)
(32), tal como fue señalado en otras ocasiones, motivo por el cual pensamos que las decisiones sobre políticas y programas laborales colombianos debieran basarse en nuestros hallazgos y no en los valores foráneos $(5,11,13)$.

En los valores de colinesterasa correspondientes a los trabajadores del valle de Aburrá y el cercano oriente de Antioquia, nosotros hemos encontrado correlación significativa tanto entre las técnicas cuantitativas para colinesterasa eritrocitaria (19) como plasmática (Carmona-Fonseca, en proceso de publicación); ahora hemos hallado que hay una alta asociación de tipo correlación entre las técnicas cuantitativas para colinesterasa eritrocitaria o plasmática y la técnica semicuantitativa tintométrica $\left(\right.$ Lovibond $^{\circledR}$ ), lo que permitirá estimar valores de unas en función de otras y viceversa. La relación entre los valores de actividad colinesterásica medida por el método tintométrico y el de Michel también ha sido informada por otros autores como buena (26), pero fue este trabajo el que criticaron McConnell y Magnotti por no haber informado sobre la concordancia de las técnicas en trabajadores agrícolas de Haití con colinesterasas levemente reducidas (25); ellos también encontraron buena concordancia entre la técnica tintométrica y el

Cuadro 4. Colinesterasas eritrocitarias y plasmáticas, medidas con la técnica de Michel (deltas $\mathrm{pH} / \mathrm{hora}$ ), según varios autores.

\begin{tabular}{|c|c|c|c|c|c|c|}
\hline \multirow{3}{*}{$\begin{array}{l}\text { autor } \\
\text { Michel } 1949\end{array}$} & \multicolumn{4}{|c|}{ Eritrocitos } & \multicolumn{2}{|c|}{ Plasma } \\
\hline & \multirow{2}{*}{$\begin{array}{c}\text { Sexo } \\
\text { general }\end{array}$} & Promedio & \multicolumn{2}{|c|}{$\mathbf{D E}$} & \multirow{2}{*}{$\begin{array}{r}\text { Promedio } \\
x x x\end{array}$} & \multirow[t]{2}{*}{ DE } \\
\hline & & 0,703 & $x x x(2)$ & 0,753 & & \\
\hline Rider 1957 & hombres & 0,766 & & 0,09 & 0,953 & 0,012 \\
\hline & mujeres & 0,75 & & 0,082 & 0,827 & 0,017 \\
\hline Sealey 1951 & general & 0,773 & & 0,09 & 0,92 & 0,2 \\
\hline Wolfsie\& Winter 1952 & general & 0,861 & & 0,09 & 0,912 & 0,112 \\
\hline \multirow[t]{2}{*}{ Fuemonth-Smith 1952} & hombres & $x x x$ & $x x x$ & 0,95 & & 0,24 \\
\hline & mujeres & $x x x$ & $x x x$ & & 0,78 & 0,12 \\
\hline Vorhaus y Kark 1953 & general & $x x x$ & $x x x$ & & 0,94 & 0,16 \\
\hline Siqueira 1978 & general & 0,838 & & 0,1 & 1,1 & 0,177 \\
\hline \multirow[t]{2}{*}{ Henao et al 1990} & hombres & 0,898 & & 0,108 & 0,953 & 0,159 \\
\hline & mujeres & 0,906 & & 0,88 & 0,875 & 0,138 \\
\hline
\end{tabular}

Fuente: con base en datos de la referencia 33: Gobierno de Guatemala, Organización Panamericana de la Salud (OPS)/Organización Mundial de la Salud (OMS), Agencia Danesa para el Desarrollo Internacional (DANIDA). Proyecto PLAGSALUD. Valores de referencia de la actividad de colinesterasa en la población guatemalteca. Guatemala: 2001.25 p. Serie de Investigación, No.1.

(1) Las unidades de medida de las técnicas son deltas $\mathrm{pH} / \mathrm{hora}$.

(2) $x x x$ : sin datos 
estándar usado (técnica espectrofotométrica modificada de Ellman), pero justamente, como en nuestro informe, hubo problema con los valores bajos de actividad enzimática porque la sensibilidad y la especificidad fueron menores de $75 \%$ para cada uno de los tres valores críticos de la prueba tintométrica comúnmente usados para definir anormalidad (75\%, $62,5 \%$ y $50 \%$ del control).

La mayor deficiencia de Lovibond ${ }^{\circledR}$ hallada en este análisis se refiere a su incapacidad de clasificar como valor bajo de actividad de colinesterasa, tanto eritrocitaria como plasmática, a aquéllos clasificados así por la prueba estándar (patrón) de Michel. Esta deficiencia también se halló frente a las técnicas $\mathrm{EQM}^{\circledR}$ y Monotest $^{\circledR}$. El análisis de sensibilidad-especificidad aquí efectuado no es estrictamente adecuado para evaluar la capacidad diagnóstica de Lovibond ${ }^{\circledR}$ porque no tiene sujetos expuestos a condiciones que reduzcan la actividad de colinesterasa y por eso se dijo que se trataba de una aproximación, pero no hay duda de que tal aproximación y los otros análisis realizados concuerdan para señalar que esa técnica mostró deficiencia notoria en cuanto a su capacidad de hallar los niveles bajos de enzima identificados por las técnicas cuantitativas. Esto obliga a proponer que se efectúe un sólido estudio de la capacidad diagnóstica de Lovibond ${ }^{\circledR}$ en una población expuesta a plaguicidas inhibidores de colinesterasa entre trabajadores afiliados al Seguro Social.

Resultó claro del análisis que las estimaciones de actividad enzimática máxima $(100 \%)$ en eritrocitos o plasma hechas con Lovibond ${ }^{\circledR}$ son significativamente mayores que las obtenidas directamente con las técnicas cuantitativas. Es posible que las diferencias no tengan significado clínico, a pesar de que lo tienen en términos estadísticos. En efecto, lo más probable es que no tenga expresión clínica diferente un valor de 0,8834 deltas $\mathrm{pH} /$ hora con la técnica de Michel para eritrocitos estimado según el valor de $100 \%$ de Lovibond ${ }^{\circledR}$, frente a 0,8570 calculado directamente con los datos y lo mismo debe suceder con las otras técnicas.

Existen muchos métodos y técnicas para medir las colinesterasas eritrocitaria y plasmática, así como la presente en sangre total. El método electrométrico aplicado por Michel (6) y consolidado por Rider y asociados (7) mide el pH y es la prueba de referencia o estándar para cuantificar la actividad de colinesterasa en eritrocitos y plasma (33); requiere condiciones de laboratorio y personal bien entrenado. El método espectrofométrico usado por Ellman y aplicado por la técnica de $\mathrm{EQM}^{\circledR}$ es un procedimiento colorimétrico, cinético o de punto final, moderadamente simple, requiere un laboratorista con relativamente poca destreza, es un procedimiento rápido, conveniente y confiable, tiene muy buena precisión y óptima sensibilidad, es más rápido y sensible que el Michel y la versión desarrollada en 1978 por la OMS con miniespectrofotómetro es portátil, sirve para trabajo de campo y conserva todas las ventajas enunciadas $(8-10,27)$. El método espectrofotométrico empleado en la técnica de Monotest ${ }^{\circledR}$ de Boehringer Mannheim (12) es un procedimiento colorimétrico cinético por variación del $\mathrm{pH}$, del que se tiene menos conocimiento en Colombia y en América en general, según los resultados de la búsqueda en la base Lilacs (Bireme, Brasil; 11 noviembre 2006); su empleo ha estado concentrado en Europa. Se trata de una técnica que es posible trabajar con equipos automatizados, como los analizadores Hitachi 737 y Cobas Bio, que usan reactivos diferentes (Hitachi 737: Boehringer Mannheim Automated Analysis for BM/Hitachi Systems; Cobas Bio: Boehringer Mannheim Monotest) (34); la automatización permite esperar resultados más homogéneos, reproducibles, procesamiento más rápidos y mayor cantidad de muestras por unidad de tiempo, con menores costos por unidad.

Hay informes sobre la conversión de unidades de tres métodos para medir colinesterasas en sangre (métodos de Ellman para sangre total y plasma, de Michel para plasma y eritrocitos y de Edson para sangre total) (35); también se sabe de otro informe sobre conversión de resultados de tres técnicas para colinesterasa en plasma (técnica con analizador de DuPont, técnica "ChE-tel" de Pfizer y técnica de Michel) (36). Otros datos conocidos son: a) conversión de unidades de actividad enzimática de colinesterasa medida por 
las técnicas manual de Michel y automática (37); b) conversión de mediciones de colinesterasa por las técnicas de Michel y de Garry y Routh (técnica de DTNB) (38); c) correlación entre valores obtenidos por técnicas manual y automatizadas (39). Todos estos informes señalan la posibilidad y la conveniencia de disponer de estos modelos para efectuar tales intercambios, lo cual debe hacerse para condiciones específicas de laboratorio, es decir, teniendo en cuenta las condiciones aplicadas al realizar la medición química.

Conviene recordar que los modelos matemáticos descritos para la conversión son valederos en las condiciones en que se midió la actividad enzimática; si tales condiciones cambian, también habrá una variación en el comportamiento de tal actividad y, en consecuencia, en los datos obtenidos. En cada uno de los artículos publicados hemos descrito con detalle las condiciones en que se hicieron nuestras mediciones $(5,11,13)$.

En conclusión, parece posible estimar en forma adecuada los valores de colinesterasa eritrocitaria o plasmática que corresponden a las técnicas cuantitativas de Michel y $\mathrm{EQM}^{\circledR}$ a partir de datos de la técnica semicuantitativa de Lovibond ${ }^{\circledR}$, y viceversa, pero sólo cuando la actividad de colinesterasa es normal, porque parece que Lovibond $^{\circledR}$ tiene muy deficiente capacidad para identificar como "bajos" los valores que realmente lo son según las pruebas cuantitativas. En la práctica epidemiológica, particularmente en el área de salud ocupacional, la medición de la actividad de colinesterasa de las personas expuestas a condiciones que pueden reducir peligrosamente sus niveles de actividad enzimática, se hace frecuentemente con una técnica de tamizaje como Lovibond $^{\circledR}$. Según nuestros hallazgos, si Lovibond ${ }^{\circledR}$ diese un resultado que indicase menos de $75 \%$ de actividad, eso sería corroborado con la técnica cuantitativa si se midiese la enzima plasmática, pero no habría concordancia si se midiese la actividad eritrocitaria, que es la que debe evaluarse en las condiciones de exposición prolongada a bajas dosis de inhibidores de colinesterasa, que son las comunes en los ambientes laborales. Pero lo más importante es que si la actividad de colinesterasa es baja según una técnica cuantitativa para eritrocitos o plasma, Lovibond ${ }^{\circledR}$ dirá en más de $60 \%$ de las veces que es adecuada (que hay más de $75 \%$ de actividad), lo que llevaría a no alejar a la persona del ambiente de riesgo. Todo esto obliga a decir que es necesario evaluar, en futuros trabajos, la capacidad diagnóstica de Lovibond ${ }^{\circledR}$ en nuestras condiciones, para definir si es aconsejable o no seguir con el uso de esta técnica.

\section{Conflictos de interés}

Ninguno para declarar.

\section{Financiación}

Se agradece al Fondo de Promoción de la Salud Industrial del Seguro Social, a la Administradora de Riesgos Profesionales Seguro Social seccional Antioquia y a la Universidad de Antioquia por la financiación del proyecto.

\section{Referencias}

1. Henao S, Zapata FM, Restrepo MP, Marín LE, Ramírez H, Corrales R, et al. Actividad colinesterásica en menores trabajadores. Antioquia (Colombia), 19891990. Medellín: Seguro Social (Administradora de Riesgos Profesionales) y Universidad de Antioquia; 1990. p.115.

2. Tintometer. Lovibond. The Lovibond cholinesterase test kit AF 267 (40-2670). Instruction. Virginia: Tintometer; 1996.

3. Thomas LC, Chamberlain GJ, Schute C. The rapid field determination of cholinesterase. En: Colorimetric chemical analytical methods of the Tintometer Company. 9th ed. New York: John Wiley \& Sons; 1980. p.397-400.

4. Limperos G, Ranta KE. A rapid screening test for the determination of the approximate cholinesterase activity of human blood. Science. 1953;117:453-5.

5. Carmona-Fonseca J, Henao S, Garcés R. Valores de referencia de actividad colinesterásica sanguínea en población laboral activa no expuesta a plaguicidas inhibidores de colinesterasa. Rev Fac Nal Salud Publ (Medellín). 2000;18:55-72.

6. Michel HO. An electrometric method for determination of red blood cell and plasma cholinesterase activity. $\mathrm{J}$ Lab Clin Med. 1949;34:1564-8.

7. Rider JA, Hodges JL Jr, Swader J, Wiggins AD. Plasma and cell cholinesterase in 800 "healthy" blood donors. J Lab Clin Med. 1957;50:376-83.

8. EQM Research Inc. (Cincinnati, Ohio, USA). Cholinesterase kit for the field determination of pesticide exposure. Instruction manual. Cincinnati: EQM; 1994. 
9. Magnotti RA Jr, Eberly JP, Quarm DE, McConell RS. Measurement of acetylcholinesterase in erythrocytes in the field. Clin Chem. 1987;33:1731-5.

10. Magnotti RA Jr, Dowling K, Eberly JP, McConnell RS. Field measurement of plasma and erythrocytes cholinesterases. Clin Chem Acta. 1988;176:315-32.

11. Carmona-Fonseca J. Valores de referencia de la actividad de la colinesterasa eritrocitaria según las técnicas de Michel y $\mathrm{EQM}^{\circledR}$ en población laboral de Antioquia, Colombia. Rev Panam Salud Pública. 2003;14:316-21.

12. den Blaauwen DH, Poppe WA, Tritschler W. Cholinesterase (EC 3.1.1.8) with butyrylthiocholine-iodide as substrate: references depending on age and sex with special reference to hormonal effects and pregnancy. J Clin Chem Clin Biochem. 1983;21:381-6.

13. Carmona-Fonseca J. Valores de referencia de colinesterasa plasmática con los métodos de Michel, $\mathrm{EQM}^{\circledR}$ y Monotest ${ }^{\circledR}$ en población laboral activa del departamento de Antioquia, Colombia. Biomédica. 2003;23:437-55.

14. Carmona-Fonseca J. Valores de colinesterasas en trabajadores activas embarazadas, menstruantes, usuarias de anticonceptivos o menopáusicas. Rev Col Obstet Ginec. 2003;54:146-56.

15. Carmona-Fonseca J. Colinesterasas eritrocitaria y plasmática en trabajadores con enfermedades crónicas controladas y en usuarios de medicamentos. Iatreia. 2006;19:14-28.

16. Carmona-Fonseca J. Valores de referencia de hemoglobina y hematocrito en una población laboral colombiana. Acta Med Colom. 2003;28:63-70.

17. Carmona-Fonseca J. Frecuencia de los grupos sanguíneos $A B O$ y $R h$ en la población laboral del valle de Aburrá y del cercano oriente de Antioquia (Colombia). Acta Med Colomb. 2006;31:20-30.

18. Carmona-Fonseca J. Relación entre los niveles de colinesterasa y los grupos sanguíneos $\mathrm{ABO}$ y $\mathrm{Rh}$. Acta Med Colomb. 2006;31:104-12.

19. Carmona-Fonseca J. Correlación e interconversión entre valores de colinesterasa eritrocitaria medida por las técnicas de Michel y EQM ${ }^{\circledR}$. Biomédica. 2006;26:53544.

20. Varona M, Morales L, Ortiz J, Sánchez José F, Cárdenas O, De la Hoz F. Panorama epidemiológico de exposición a plaguicidas inhibidores de colinesterasa en 17 departamentos del país. Biomédica. 1998;18:229.

21. Silva E. Morales L, Ortiz J. Evaluación epidemiológica de plaguicidas inhibidores de acetilcolinesterasa en Colombia, 1996-1997. Biomédica. 2000;20:200-9.

22. Cárdenas O, Silva E, Morales L, Ortiz J. Estudio epidemiológico de exposición a plaguicidas organo- fosforados y carbamatos en siete departamentos colombianos, 1998-2001. Biomédica. 2005;25:170-80.

23. Idrovo AJ. Vigilancia de las intoxicaciones con plaguicidas en Colombia. Rev Salud Pública. 2000;2. [Consultado: 11 de noviembre 2006]. Disponible en: http://www.revmed.unal.edu.co/revistasp/v2n1/ Rev213.htm.

24. Edson EF. Blood tests for users of OP insecticides World Crops. 1950;10:49-51.

25. McConnell R, Magnotti R. Screening for insecticide overexposure under field conditions: a reevaluation of the tintometric cholinesterase kit. Am J Public Health. 1994;84:479-81.

26. Miller S, Shah MA. Cholinesterase activities of workers exposed to organophosphorus insecticides in Pakistan and Haiti and an evaluation of the tintometric method. J Environ Sci Health B. 1982;17:125-42.

27. Ellman GL, Courtney KD, Andres V Jr, FeatherStone RM. A new rapid colorimetric determination of acetylcholinesterase activity. Biochem Pharmacol. 1961;7:88-95.

28. McConnell R, Cedillo L, Keifer M, Palomo MR. Monitoring organophosphate insecticide exposed workers for cholinesterase depression: new technology for office or field use. J Occup Med. 1992;34:34-7.

29. Departamento de Salud Ocupacional y Contaminación Ambiental, Instituto de Salud Pública de Chile. Protocolo. Examen de salud para aplicaciones de plaguicidas. Versión 2004. [Consultado: 11 de noviembre 2006]. Disponible en: http://www.ispch.cl/ salud_ocup/doc/diagrama_datos.doc.

30. Da Silva ES, Midio AF, Garcia EG. A field method for the determination of whole blood cholinesterase. Med Lav. 1994;85:249-54.

31. Silva JJ. A utilização das colinesterases na avaliação da exposição humana a agrotóxicos. Novas perspectivas para antigas ferramentas (tese). São Paulo: Universidade de São Paulo; 2004. p.100.

32. Gobierno de Guatemala, Organización Panamericana de la Salud (OPS)/Organización Mundial de la Salud (OMS), Agencia Danesa para el Desarrollo Internacional (DANIDA). Proyecto PLAGSALUD. Valores de referencia de la actividad de colinesterasa en la población guatemalteca. Serie de Investigación No.1. Ciudad de Guatemala: Gobierno de Guatemala; 2001. p.25.

33. Henao S, Corey G. Plaguicidas inhibidores de las colinesterasas. Serie Vigilancia 11. Centro Panamericano de Ecología Humana y Salud. Metepec: ECO, OPS, OMS; 1991.

34. Krull NB, Kropf J, Gressner AM. Influence of reagent composition on atypical pseudocholinesterase activity measurement: comparison of a manual and an 
automated method and implications for routine. Eur $\mathrm{J}$ Clin Chem Clin Biochem. 1992;30:545-6.

35. Da Silva ES, Midio AF. Interconversion of units of three methods for blood cholinesterases. Med Lav. 1998;89:265-72.

36. Newman MA, Que Hee SS. Interconversion and comparison of the results of three methods for cholinesterase in serum. Clin Chem. 1984;30:308-10.

37. Groff WA, Kaminskis A, Ellin RI. Interconversion of cholinesterase enzyme activity units by the manual delta
$\mathrm{pH}$ method and a recommended automated method. Clin Toxicol. 1976;9:353-8.

38. Morse CS. Interconversion of cholinesterase measurements by the deltapH method of Michel and the DTNB method of Garry and Routh. Clin Toxicol. 1974;7:38993.

39. Grainger MM, Groff WA, Ellin RI, Arsenal E. Blood cholinesterase values. Correlation obtained by automated and manual techniques. Arch Environ Health. 1968;16:821-2. 